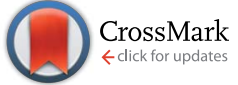

Cite this: RSC Adv., 2017, 7, 13299

\title{
Control of surface radical graft polymerization on polyester fibers by using Hansen solubility parameters as a measurement of the affinity of chemicals to materials $\uparrow$
}

\author{
Maryam Tamizifar and Gang Sun* \\ Radical graft polymerization is an effective method for surface modification of inert and hydrophobic \\ poly(ethylene terephthalate) (PET) fibers, but is lacking grafting efficiency and control. Several key \\ interactive and controlling elements of the graft polymerization reaction were identified, and \\ corresponding affinity characters of each reaction component were quantified by using Hansen solubility \\ theory. Proper control of the radical graft polymerization reaction on PET fiber surfaces was achieved.
}

Received 23rd November 2016 Accepted 20th February 2017

rsc.li/rsc-advances

in the total mass. In order to achieve desired functions on the

Poly(ethylene terephthalate) (PET) fibers have hydrophobic behavior, low surface energy, and poor wettability because of the lack of polar groups in their structures. Surface modification of PET fibers can improve and alter properties or introduce new functionalities such as hydrophilicity, super-hydrophobicity, biocompatibility, conductivity, dyeability, anti-fouling, and antibacterial properties to the fibers. ${ }^{1-5}$ Although plasma treatment has been a successful method for surface treatment of PET, it requires complex and expensive operating devices. ${ }^{6-9}$ Also the relatively wide energy range $(0.5-5 \mathrm{eV})$ of the glow discharge generates a large number of charged and neutral molecular fragments, making the control of the reaction mechanism difficult. Radical graft polymerization has been considered as a possible and effective method for surface modification of inert polymers where various functional vinyl monomers can be incorporated. ${ }^{10-15}$ The most common initiation process is thermal decomposition of initiators through homolytic cleavage of weak covalent bonds in the molecule to free radicals. These initiators mainly fall into peroxy molecules due to the structural features and tendency in abstracting active hydrogens on polymers. Upon heating to its decomposition temperature, the weak $\mathrm{O}-\mathrm{O}$ bond in alkyl peroxide initiator ( $\left.\mathrm{RO}^{-} \mathrm{OR}^{\prime}\right)$ forms two highly active $\mathrm{RO}^{\bullet}$ radicals, which have high energy and preference to abstract $\mathrm{H}$-atom from a polymer substrate (PET-H). Due to formation of a strong bond (RO-H), such a reaction path will reduce the overall Gibbs free energy of the reaction $(\Delta G<0)$, making it a thermodynamically favorable reaction versus direct radical addition reaction to vinyl monomers. Fibers have limited surface areas, normally less than $1 \%$ by weight

Fiber and Polymer Science, Division of Textiles and Clothing, University of California, Davis, CA 95616, USA. E-mail: gysun@ucdavis.edu; Fax: +1 530752 7584; Tel: +1 530 7520840

$\dagger$ Electronic supplementary information (ESI) available: The grafting procedure and Hansen solubility parameter information of chemicals and materials used. See DOI: $10.1039 / \mathrm{c} 6 \mathrm{ra} 27186 \mathrm{c}$ chemically modified fibers, high yields of surface grafting are absolutely necessary. Thus, having proper control on the radical graft polymerization reaction on fiber surfaces is significantly important to increase the grafting reaction yields. According to desired graft polymerization reaction conditions, it is crucial to have all related reactants (initiator and monomer) at very close proximity of the PET surface. Thus, high affinity of the molecules (initiator and monomers) toward PET is essential, which could increase their accessibility to the polymer chains and facilitate abstraction of $\mathrm{H}$-atom from and subsequent grafting of functional monomers on the PET surfaces. Based on energy distributions of all possible bonds in PET, we speculate that the $\mathrm{H}$-abstraction reaction mostly occurs on $\mathrm{CH}_{2}$ groups of ethylene glycol component. In addition, the reagents should be well diffused into fiber surfaces for intimate molecular contacts and improved reaction efficiency. With all conditions mentioned, an organic solvent that can swell PET and facilitate diffusion of initiators and monomers into the polymer might be necessary in assisting the grafting reaction. A small amount of such solvent was proposed to be added in formulations of treatment systems and could be removed and possibly recycled during the treatment of the fabrics. Thus, affinity of the solvent to the initiator, monomer and PET is important and should be considered as well. In this study, Hansen solubility parameters (HSP) was used as a tool to quantify affinity of initiators, solvents, and monomer molecules to PET. ${ }^{16}$ The initiations of radicals and grafting polymerization were accomplished at a curing process under high temperatures (above glass temperature of PET) in an oven on PET fabrics where the initiator, solvent, and monomers were evenly loaded. The curing of the fabrics under the elevated temperatures ensured swelling of the fibers by the selected solvent, increased diffusion of the initiator and monomers into the fibers and subsequent evaporation of the solvents $\left(\mathrm{H}_{2} \mathrm{O}\right.$ and the organic solvent). Diffused initiator in the 
PET fibers could guarantee effective abstraction of $\mathrm{H}^{\bullet}$ from weak $\mathrm{C}-\mathrm{H}$ bonds of PET when the temperature prompts the formation of initiator radicals. Different studies have shown that if nucleophilicity of the attacking radical increases, it would favor hydrogen abstraction rather than addition to an unsaturated system (benzene ring or double bond). ${ }^{\mathbf{1 7 - 1 9}}$ The resulted PET $^{*}$ radicals can further react with monomers, leading to grafting and avoiding undesirable homo-polymerization of the monomers (Scheme 1).

Hansen solubility theory (HSP) and more specifically Hansen solubility parameter distances between chemicals, " $R$ " values, were employed in measurements of affinities among these chemicals and polymers (ESI material $\dagger$ ). Different from Hildebrand solubility theory, HSP considers comprehensive intermolecular interactions, including dispersion forces, polar interactions, and hydrogen bonds, and can even work on complex systems such as protein solutions..$^{20}$ Four different initiators, four organic solvents, and two monomers were selected in the study, which have varied HSP distances to PET and could affect their affinity and reactivity to PET (ESI Tables 2-4†). First, successful graft polymerization reactions on PET surfaces were demonstrated by using two selected monomers, tetramethyl-4-piperidinyl methacrylate (TMPM) and 2acrylamido-2-methyl-1-propane sulfonic acid (AMPS), working with four initiators, benzoyl peroxide (BPO), tert-butyl proxy benzoate $(t \mathrm{BPB})$, di-cumyl peroxide (DCP), and di-tert-butyl peroxide (DtBP), in the same solvent of ethyl benzoate (EB), respectively, and the structures of the products were confirmed by Fourier transform infrared (FTIR). $\uparrow$ As described in ESI materials, all chemicals were emulsified and evenly applied onto a piece of PET fabric by padding with a controlled pressure. The samples were cured in an oven to the designed temperatures for a desired time (ESI Table $2 \dagger$ ). Under the heating, the fabrics became completely dry, any residual solvent should have been completely removed, and monomers should have been incorporated onto the a)

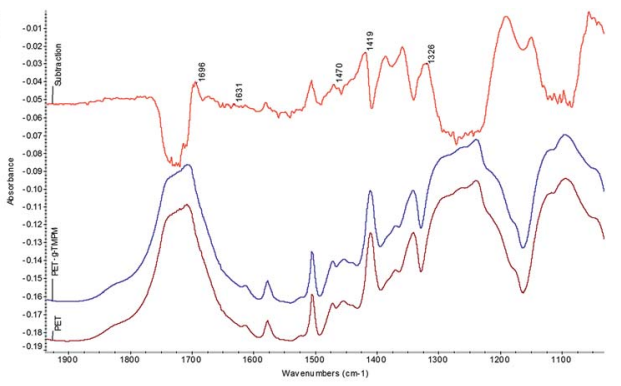

b)

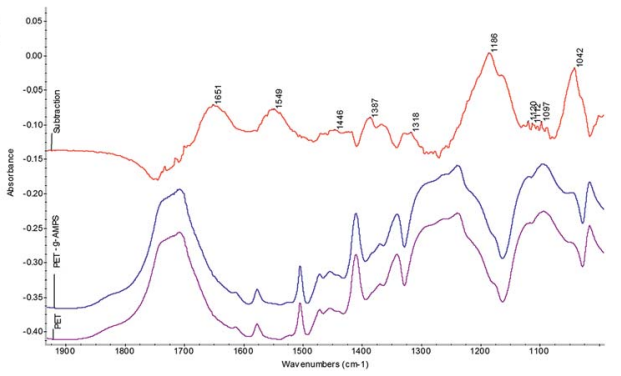

Fig. 1 FTIR spectra of the original PET, grafted samples with (a) TMPM and (b) AMPS as monomers, and corresponding subtraction spectra using $\mathrm{BPO}$ as an initiator and $\mathrm{EB}$ as a solvent.

polymer. And then the fabrics were consequently washed thoroughly to remove any unreacted chemicals and ready for subsequent measurements. Fig. 1(a and b) shows FTIR spectra of the grafted samples from two monomers (PET-g-monomer) with BPO as an initiator and EB as a solvent. TMPM is a monomer with an HSP distance to PET $\left(R(\mathrm{HSP})_{\text {TMPM-PET }}=10\right)$, while AMPS has $R(\mathrm{HSP})_{\mathrm{AMPS}-\mathrm{PET}}=16$. A subtraction spectrum of PET- $g$-TMPM by PET does show bands at $1696 \mathrm{~cm}^{-1}$ for $\mathrm{C}=\mathrm{O}$ stretch and at 1189 and $1150 \mathrm{~cm}^{-1}$ for CO-O stretching of ester group in TMPM structure (Fig. 1(a)). The subtraction spectrum of PET- $g$-AMPS

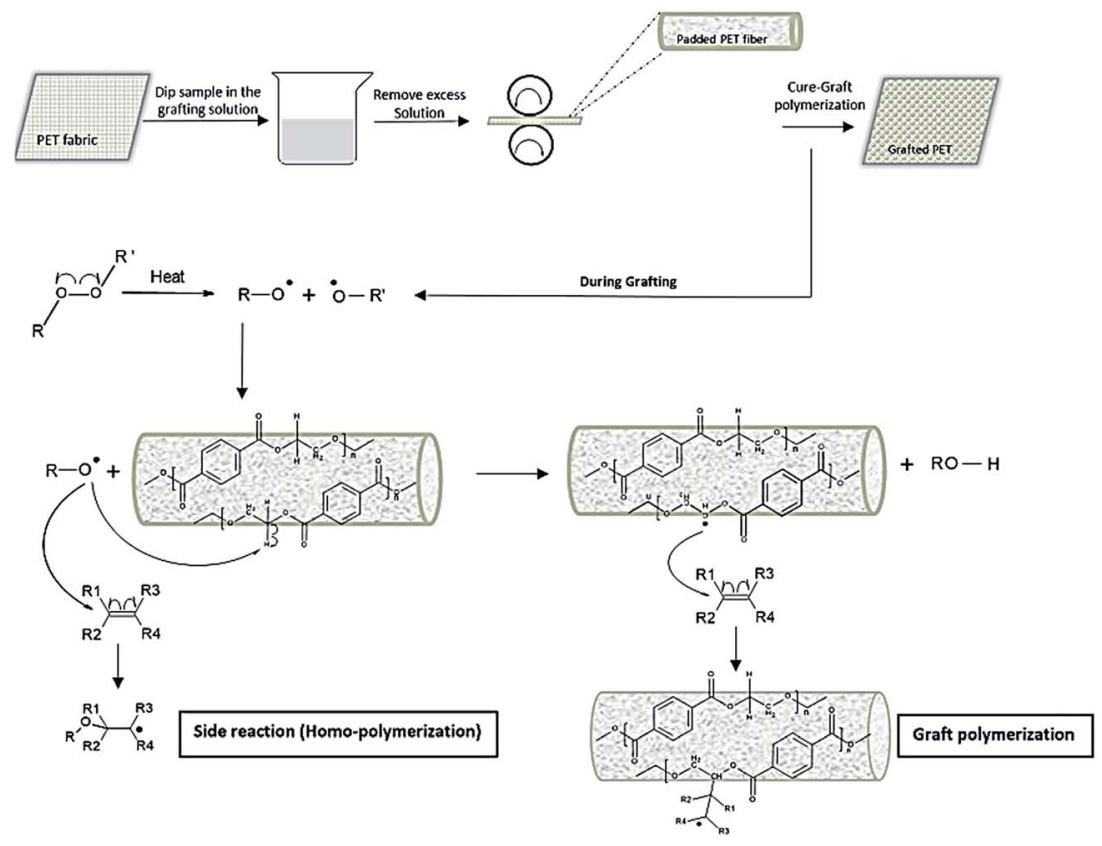

Scheme 1 Overall treatment process and proposed mechanisms of radical reactions. 
shows characteristic bands of amide I, amide II, asymmetric and symmetric $\mathrm{S}=\mathrm{O}$ stretching at 1651, 1549, 1186, and $1042 \mathrm{~cm}^{-1}$, respectively (Fig. 1(b)).

Three additional initiators were selected, and HSP distances of them to PET and two monomers were calculated and are shown in Table 1. The graft polymerizations of TMPM and AMPS on PET surfaces using the initiators were conducted, and the grafting yields and moisture regains of the grafted products are depicted in Fig. 2(a and b). Here, based on speculations on surface modification on PET, initiator and monomer should both be close to PET. Among four initiators, BPO, with a HSP distance to PET $R(\mathrm{HSP})_{\mathrm{BPO}-\mathrm{PET}}=2.1$, is the closest one to PET, meaning having the best affinity to the fiber, showed relatively higher grafting yields than that of other initiators. Also this HSP distance $\left(R_{\mathrm{BPO}-\mathrm{PET}}=2.1\right)$ is lower than the distance of $\mathrm{BPO}$ to EB as a solvent $\left(R_{\mathrm{BPO}-\mathrm{EB}}=4.2\right)$ unlike other three initiators. This implies that BPO, having lower affinity to solvent (EB), would like to avoid EB and move closer to PET surface than other initiators, consequently generating more polymer radicals. Thus, BPO should present the highest grafting efficiency among all initiators, consistent to the results (Fig. 2(a and b)).

TMPM has a better affinity to BPO $\left(R(\mathrm{HSP})_{\mathrm{TMPM}-\mathrm{BPO}}=8.4\right)$ than to PET polymer $\left(R(\mathrm{HSP})_{\text {TMPM-PET }}=10\right)$, and thus is easier for BPO to approach and attack it directly, leading to

Table 1 The mutual HSP distances of monomers, initiators, solvents and PET

\begin{tabular}{lcclllll}
\hline & PET & EB & NMP & BS & Tol & TMPM & AMPS \\
\hline PET & 0 & 6 & 2.8 & 5.9 & 9.2 & 10 & 16 \\
BPO & 2.1 & 4.2 & 2.7 & 4.5 & 7.2 & 8.4 & 17 \\
$t$ BPB & 7 & 4.3 & - & - & - & 6 & 16.5 \\
DCP & 9.3 & 3.8 & - & - & - & 3.5 & 20.6 \\
D $t$ BP & 12 & 7.5 & - & - & - & 5 & 20.7 \\
TMPM & 10 & 4.3 & 8.7 & 6.9 & 3 & 0 & - \\
AMPS & 16 & 19.6 & 15 & 16.1 & 22.2 & - & 0
\end{tabular}
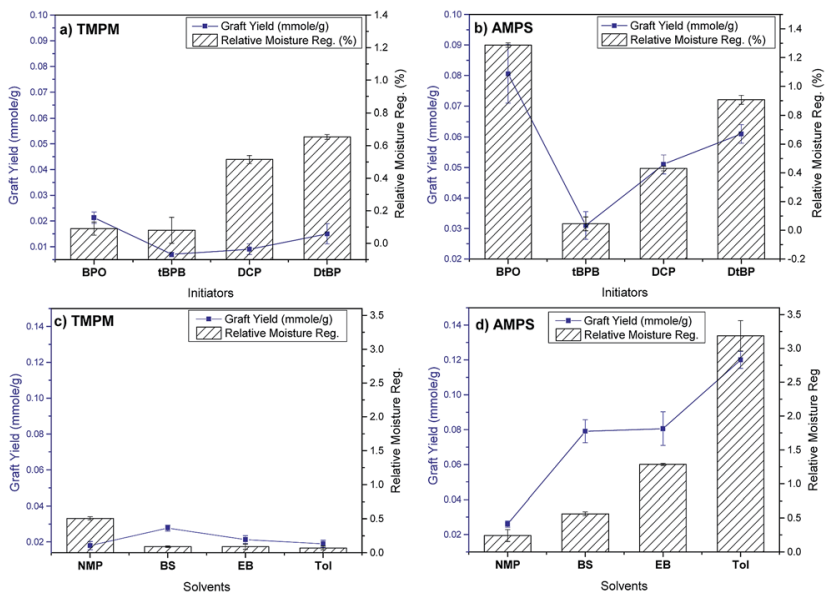

Fig. 2 Grafting yields and moisture regains of grafted samples with different initiators (EB as a solvent) for (a) TMPM, (b) for AMPS monomer, and using different solvents (BPO as initiator) for (c) TMPM, (d) for AMPS monomer. homopolymerization rather than graft polymerization of TMPM on PET polymer, a reason for the lower grafting yield compared to the PET- $g$-AMPS. Furthermore, TMPM monomer is closer to the EB than to PET $\left(R(\mathrm{HSP})_{\mathrm{TMPM}-\mathrm{PET}}=10\right)$ and $\left(R(\mathrm{HSP})_{\mathrm{TMPM}-\mathrm{EB}}\right.$ $=4.3$ ) reflecting the fact that TMPM has higher tendency to remain in the solvent than being attracted towards PET surface, possibly leading to radical transfer reaction to the solvent and early termination of the polymerizations. AMPS monomer is closer to PET than to $\mathrm{BPO}$ and $\mathrm{EB}\left(R(\mathrm{HSP})_{\mathrm{AMPS}-\mathrm{PET}}=16\right.$, $R(\mathrm{HSP})_{\mathrm{AMPS}-\mathrm{BPO}}=17$, and $\left.R(\mathrm{HSP})_{\mathrm{AMPS}-\mathrm{EB}}=19.6\right)$ according to HSP distances, which could favor its graft polymerization on PET than interacting with the solvent, generating higher grafting values (Fig. 2). In fact, all other three initiators are similar to BPO, having closer HSP distances to PET than to AMPS (Table 1), leading to the overall better grafting yields of AMPS than those of TMPM onto PET fibers. Among these three initiators, $t \mathrm{BPB}$ is the least efficient one since it produces one benzoyloxy radical and one alkoxide radical, which are not equally reactive to abstraction of hydrogen atoms from the polymer. ${ }^{21}$ Thus, it is less effective in initiating the reactions on PET, and a lower grafting yield was observed on the use of TMPM as a monomer.

The trends of graft polymerizations of TMPM and AMPS on PET using these four initiators were fully predictable according to Hansen solubility parameters, providing the solid evidence in control of the reactions. To continue the control of the reactions, the addition of selected organic solvents was explored by using the HSP theory.

Here, organic solvents, $N$-methyl-2-pyrrolidone (NMP), $N$-butyl salicylate (BS), and toluene (Tol), in addition to ethyl benzoate (EB), were employed to assist swelling of PET and diffusion of initiators and monomers into PET. ${ }^{22-25}$ The affinities of these solvents to PET, BPO and two monomers are listed in Table 1 as well. NMP has the lowest HSP distance to PET $\left(R(\mathrm{HSP})_{\mathrm{NMP}-\mathrm{PET}}=2.8\right)$, and Tol has the largest distance $\left(R(\mathrm{HSP})_{\text {Tol-PET }}=9.2\right)$. The order of the affinity of solvents to PET is NMP $>\mathrm{BS}>\mathrm{EB}>\mathrm{Tol}$, and affinity of solvents to BPO is NMP $>$ $\mathrm{EB}>\mathrm{BS}>$ Tol, expecting increasing grafting values from solvent NMP to Tol which is the case for AMPS (Fig. 2(c and d)). For AMPS the $R(\mathrm{HSP})_{\mathrm{AMPS}-\text { solvent }}$ increases from NMP to Tol (from 15 to 22.2), concluding that AMPS has decreasing affinity to the solvent from NMP to BS, EB and Tol. Therefore, the AMPS monomer is driven to PET rather than remaining in the solvent, resulting in increased probability of reacting with PET $^{*}$ and yielding higher grafting values. It is noteworthy of stating that for AMPS both the initiator (BPO) and the monomer have similar increasing trend of HSP distances toward solvents from NMP to Tol. This may provide an additive effect on the grafting reaction of this monomer to PET compared to TMPM where only the $R(\mathrm{HSP})_{\mathrm{BPO}-\text { solvent }}$ has the increasing trend. For TMPM the $R(\mathrm{HSP})_{\text {TMPM-PET }}=10$ is higher than $R(\mathrm{HSP})_{\text {TMPM-solvent }}(8.7$, 6.9, 4.3, 3 for NMP, BS, EB and Tol, respectively) meaning that the TMPM monomer prefers to remain in the solvent phase rather than migrating or diffuse to PET surfaces and react with $\mathrm{PET}^{*}$, leading to overall lower grafting values.

The monomer TMPM showed quite low and close grafting yields (in different solvents), making the individual analysis difficult. The overall results revealed quite consistent 
relationships of HSP distances to the affinity of the chemicals in the environments and the grafting efficiency, thus, leading us to believe the control of graft polymerizations on PET fibers could be achieved.

As an additional supporting evidence, moisture regains of the grafted samples were also measured. The AMPS grafted PET samples presented consistent moisture regains with the grafting yields, which is due to the hydrophilicity of the monomer. However the TMPM grafted samples revealed slight inconsistency in moisture regains and grafting yields, possibly caused by the hydrophobic nature of TMPM.

To further confirm the grafted products, scanning electron microscope (SEM) was employed to examine surface morphologies of the control, blank (sample grafted with no monomer in EB solvent using BPO as an initiator), and grafted PET samples with highest grafting yields (Fig. $3(\mathrm{a}-\mathrm{e})$ ). $\dagger$ No significant morphological change was observed for the blank sample (Fig. 3(b)) that had been treated with the same condition without a monomer. For the sample grafted with AMPS monomer (Fig. 3(d and e)) definite changes of surface structural features can be observed with polymer chains grafted and coalesced onto the fiber surface. For TMPM grafted sample less morphological change is observed with scattered grafted polymer chains coating the PET surfaces (Fig. 3(c)). These results confirmed that the radical graft polymerization of different monomers onto PET surfaces was successfully achieved and surface morphologies varied by different monomers.

The surface elemental analyses of the grafted samples were characterized by energy dispersive X-ray spectroscopy (EDS) at 5 $\mathrm{kV}$ acceleration voltages with gold coating (Fig. 4). Red labelled regions represent carbon (C), green represents oxygen and blue represents sulfur (S). It can be seen that for the original PET sample only $\mathrm{O}$ and $\mathrm{C}$ were detected (Fig. 4(a)) and for the blank sample green regions (representing $\mathrm{O}$ ) were increased compared to the original sample due to oxidation of PET fibers by the initiator in absence of a monomer (Fig. 4(b)). For the sample grafted with TMPM red (representing C) regions are more detectable which could be related to high number of carbons in the TMPM monomer structure confirming its successful grafting on PET surfaces (Fig. 4(c)). For the sample grafted with AMPS more green labeled area $(\mathrm{O})$ and dispersed blue regions (representing S) are detected compared to the
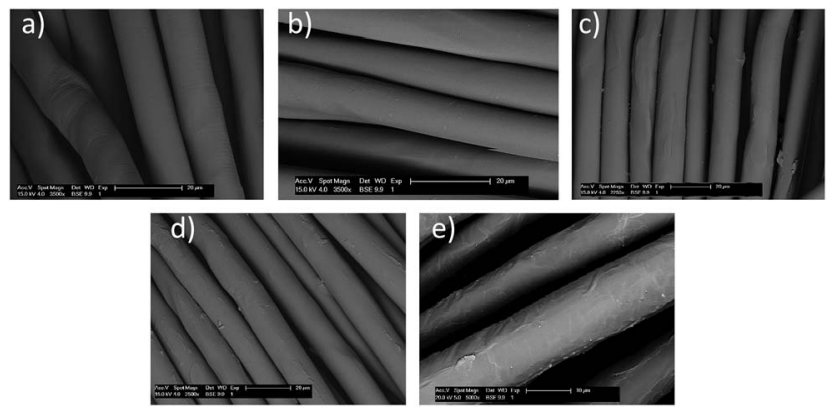

Fig. 3 SEM images of PET grafted samples: (a) raw PET, (b) blank PET, (c) PET grafted with TMPM, and (d and e) PET grafted with AMPS (BPO as initiator and EB as solvent).
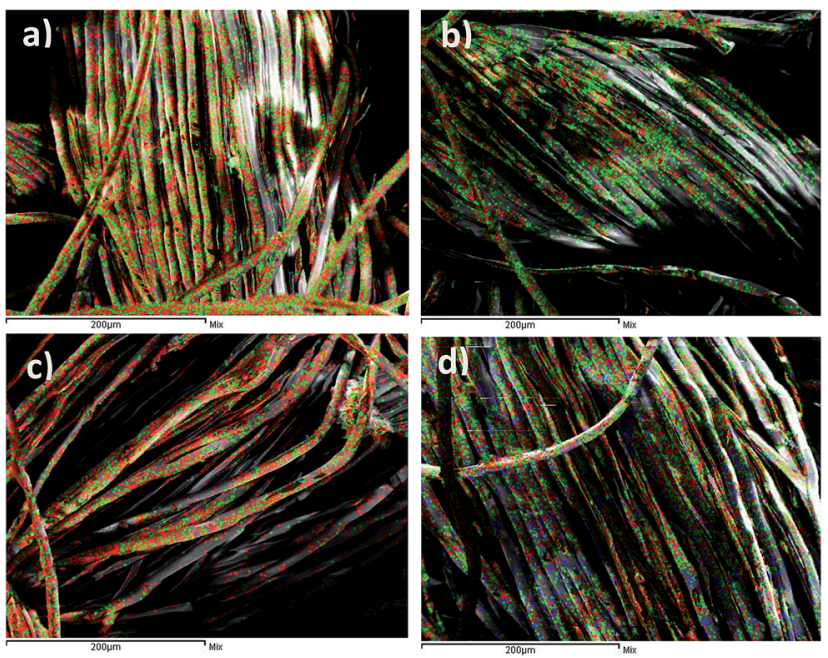

Fig. 4 Elemental analysis of the (a) original PET, (b) blank PET, (c) grafted sample with TMPM and (d) grafted sample with AMPS using $\mathrm{BPO}$ and EB as the initiator and solvent, respectively.

original PET, confirming successful grafting of AMPS on PET surfaces (Fig. 4(d)). It should be noted that no nitrogen (N) signal was detected in both TMPM or AMPS grafted samples because of its close atomic number with carbon. $\dagger$

Since the AMPS grafted samples contain anionic sulfonic groups which can be employed in dyeing with a cationic dye, methylene blue. The dyeing exhaustion (or uptake, $E \%$ ), $K / S$ values and surface microscope images of control and dyed PET$g$-AMPS samples are shown in Fig. 5(a-d). As expected, very low dye exhaust, $K / S$ value, and very light blue shade of the untreated (raw) PET fabric were observed since PET does not have much reactive sites with cationic dyes. Similarly, the blank sample also showed low values of dye exhaust and $K / S$, clarifying the fact that any dye uptake by the fiber is a result of ionic interactions.

A significant increase in both $E \%$ and $K / S$ was observed for the grafted samples with different initiators and solvents (Fig. 5(a and b)). This noticeable increase can be attributed to the anionic sulfonic groups introduced by the radical graft polymerization of AMPS monomer. For the samples grafted

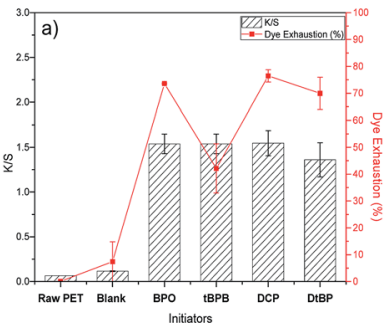

c)

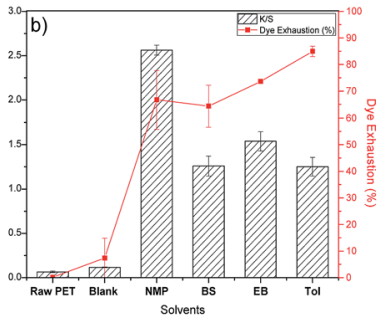

d)
Fig. $5 \mathrm{~K} / \mathrm{S}$, dye exhaustion values, and microscopic images of AMPS grafted samples dyed with methylene blue dye for (a and $c$ ) different initiators (BPO, tBPB, DCP, DtBP) and ( $b$ and d) different solvents (NMP, $B S, E B, T o l)$. 
using different initiators (Fig. 5(a)) the $K / S$ values for all initiators seem to be very close with slight decrease for $\mathrm{D} t \mathrm{BP}$ as an initiator, whereas the $E \%$ values have almost the same trend as the grafting values for AMPS grafted samples (Fig. 2(b)). For the samples grafted under different solvents the exhaustion values have the expected increasing trend from NMP to Tol, consistent with the affinity results, except that NMP has an exceptional high value. The exceptional high value from NMP is possibly due to its very low HSP distance to PET where it can swell the polymer and possibly increase amorphous regions in the fibers, which could possibly result in more dye exhaustion. Therefore, such an increase is not related to the grafting of AMPS on the PET fibers. For the samples grafted in other solvents the dye seems to stain locally the grafted areas on the surface not as uniform as the sample grafted in NMP as a solvent. Overall it seems like $E$ \% values better reflect surface ionic interactions of dye molecules and grafted samples which could justify the results obtained from dyeing the grafted samples using different initiators and solvents.

\section{Conclusion}

In a novel approach to have control on the surface radical graft polymerization of PET surfaces Hansen solubility theory was utilized to analyze factors and affinities of initiators, monomers and solvents to PET. Hansen solubility parameter distance $R$ (HSP) was proven as a tool to quantify affinity between different components of the grafting reaction and provide selection of solvents and initiators with suitable affinities to monomers and PET polymer. It appears that four major distances, initiator-PET, initiator-solvent, monomer-PET, and monomer-solvent, in the grafting reaction system have major roles in the fate and result of the grafting reaction efficiency.

\section{Acknowledgements}

M. Tamizifar acknowledges the Jastro Shields graduate student research awards from the University of California, Davis.

\section{References}

1 M. Ciobanu, A. Siove, V. Gueguen, L. J. Gamble, D. G. Castner and V. Migonney, Biomacromolecules, 2006, 7, 755.

2 O. Sanli, R. Zemzem and H. I. Ünal, J. Macromol. Sci., Part A: Pure Appl. Chem., 2003, 40(9), 947.

3 Y. L. Hsieh and M. Wu, J. Appl. Polym. Sci., 1991, 43(11), 2067.
4 F. C. Loh, K. L. Tan, E. T. Kang, K. G. Neoh and M. Y. Pun, Eur. Polym. J., 1995, 31(5), 481.

5 R. l. Barbey, L. Lavanant, D. Paripovic, N. Schüwer, C. Sugnaux, S. Tugulu and H. Klok, Chem. Rev., 2009, 109, 5437.

6 R. Davis, A. El-Shafei and P. Hauser, Surf. Coat. Technol., 2011, 205(20), 4791.

7 J. Friedrich, W. Unger, A. Lippitz, I. Koprinarov, A. Ghode, S. Geng and G. Kuhn, Compos. Interfaces, 2003, 10, 139.

8 B. Gupta, C. Plummer, I. Bisson, P. Frey and J. Hilborn, Biomaterials, 2002, 23(3), 863.

9 J. K. Wang, X. Y. Liu and H. S. Choi, J. Polym. Sci., Part B: Polym. Phys., 2008, 46(15), 1594.

10 S. Liu and G. Sun, Polymer, 2008, 49, 5225.

11 J. H. Li, D. S. Tan, X. Q. Zhang, H. Tan, M. M. Ding, C. X. Wan and Q. Fu, Colloids Surf., B, 2010, 78, 343.

12 S. Bedel, B. Lepoittevin, L. Costa, O. Leroy, D. Dragoe, J. Bruzaud, J. M. Herry, M. Guilbaud, M. N. BellonFontaine and P. Roger, J. Polym. Sci., Part A: Polym. Chem., 2015, 53, 1975.

13 Y. Jiang, Y. Liang, Z. Y. Du, H. W. Zhang and R. Zhang, Acta Polym. Sin., 2013, 11, 1419.

14 J. Li, F. Lin, L. D. Li, J. Li and S. Liu, Macromol. Chem. Phys., 2012, 213, 2120.

15 J. D. Wu, Y. L. Jiang, J. He, S. F. Zhao, G. Q. Cai and J. P. Wang, Text. Res. J., 2016, 86, 677.

16 Hansen Solubility Parameter in Practice (v4.1.07) edn, http:// hansen-solubility.com/HSPiPPurchase.html.

17 G. Moad and H. D. Solomon, The Chemistry of Radical Polymerization, Elsevier Ltd., Oxford, UK, 2nd edn, 2006, p. 28.

18 R. A. Sheldon and J. K. Kochi, J. Am. Chem. Soc., 1970, 92(14), 4395.

19 W. A. Pryor, F. Y. Tang, R. H. Tang and D. F. Church, J. Am. Chem. Soc., 1982, 104(10), 2885.

20 A. Aghanouri and G. Sun, RSC Adv., 2015, 5, 1890.

21 A. Ravve, Principles of Polymer Chemistry, Springer Science + Business Media, LLC, New York, 1st edn, 1995, p. 35.

22 H. L. Needles and M. J. Park, J. Appl. Polym. Sci., 1996, 59, 1683.

23 O. Sanli and E. Pulat, J. Appl. Polym. Sci., 1993, 47(1), 1.

24 A. S. Ribnick, H. D. Weigmann and L. Rebenfeld, Text. Res. J., 1972, 42, 720.

25 B. H. Knox, H. D. Weigmann and M. G. Scott, Text. Res. J., 1975, 45, 203. 